\title{
Follow-Up of Patients with Breast Cancer: Imaging of Local Recurrence and Distant Metastases
}

\author{
Ulrich Bick and Thomas H. Helbich
}

\section{Learning Objectives}

- To learn about the strengths and weaknesses of the different imaging modalities for local surveillance of patients with a personal history of breast cancer.

- To understand that mammography is the only routinely recommended imaging modality with which to detect local recurrence or contralateral breast cancer.

- To discuss the role and effectiveness of imaging modalities in the staging and surveillance of patients with breast cancer after primary therapy.

- To show that there is little justification for imaging to detect metastasis in asymptomatic breast cancer patients.

\subsection{Introduction}

Breast cancer is the leading cause of cancer-related death among women worldwide, with variable incidence and mortality rates. Fortunately, mortality has decreased because of advances in screening and treatment $[1,2]$. The 10-year survival rate of breast cancer ranges from 70 to $80 \%$, with up to $90 \%$ for local and $60 \%$ for regional disease [3]. The annual hazard of recurrent disease (local and or metastases) ranges between 2 and 5\% in years 5-20 after diagnosis. The yield for recurrent disease is likely to be higher in patients with

\footnotetext{
U. Bick $(\bowtie)$

Department of Radiology, Charité - Universitätsmedizin Berlin, Berlin, Germany

e-mail: ulrich.bick@charite.de

T. H. Helbich $(\bowtie)$

Department of Biomedical Imaging and Image-guided Therapy, Division of Molecular and Gender Imaging, Medical University of Vienna, Vienna, Austria

e-mail: thomas.helbich@meduniwien.ac.at
}

advanced stages of disease [4]. Recent studies have demonstrated that the onset of local recurrence is an independent predictor for survival [5]. Thus management of patients with breast cancer during surveillance plays an important role. The aims of any follow-up are to detect early local recurrence or contralateral breast cancer and to diagnose and treat cancer and/or therapy-related diseases such as metastases and osteoporosis [3]. Considering these facts a welldefined, evidence-based surveillance protocol is needed to manage patients with breast cancer after the initial diagnosis, including staging and follow-up. Currently mammography every 1-2 years is the only recommended evidence-based imaging modality. In asymptomatic patients, there are no data to indicate that any imaging or laboratory test leads to a survival benefit. In symptomatic patients or in case of clinical findings appropriate tests should be performed immediately [3-8].

The purpose of this chapter is to address the role and effectiveness of imaging modalities in the staging and surveillance of patients with breast cancer after primary therapy.

\subsection{Surveillance Recommendations for Local Recurrence}

Local recurrence in breast cancer can be defined as recurrent disease to the ipsilateral breast, chest wall, or regional lymph nodes. Most local recurrences in breast cancer can be treated with curative intent and early detection of local recurrence will improve the overall prognosis of the patients [9]. Overall, the incidence of an ipsilateral breast recurrence (including ipsilateral new primary cancers) in women with spontaneous early-stage breast cancer treated with breast-conserving therapy will be around $0.5-1 \%$ per year and may especially in estrogen-receptor-positive tumors remain elevated well beyond 10 years after diagnosis $[9,10]$. However, a variety 
of factors will influence the likelihood and timing of local recurrence. In particular, involved surgical margins, nonadherence to adjuvant therapy guidelines, large size, and aggressive biology of the primary tumor, young patient age at diagnosis, and presence of familial or genetic risk factors may substantially increase recurrence rates.

\subsubsection{Surveillance After Breast-Conserving Surgery}

The backbone of surveillance of the ipsilateral breast in asymptomatic women with a personal history of breast cancer and who were treated with breast-conserving surgery is mammography [11-13]. However, specific recommendations vary substantially between countries and institutions regarding the optimal time to begin of mammographic surveillance after local treatment (e.g., 6-24 months after surgery) and the frequency (from semiannual mammography of the ipsilateral breast during the first 2 or 3 years after surgery to mammography only every 2-3 years) [9]. As sensitivity of mammography in the treated breast may be lower due to posttreatment changes [9], mammography is usually accompanied by a clinical exam and in many European countries by breast ultrasound [3]. This has the advantage that the axilla and regional lymph nodes can be evaluated at the same time. Digital breast tomosynthesis (DBT) has the potential to improve screening accuracy compared to 2D mammography; however data regarding DBT in the follow-up of patients with breast cancer is limited [14]. Contrast-enhanced magnetic resonance imaging (MRI) has the highest sensitivity regarding ipsilateral breast cancer recurrence. MRI is therefore an excellent tool for surveillance of asymptomatic highrisk women with a personal history of breast cancer as well as for problem-solving in otherwise indeterminate cases. However, the available evidence is currently insufficient to recommend for or against MRI as routine surveillance method in all normal-risk women with a personal history of breast cancer [15].

\subsubsection{Follow-Up After Mastectomy}

Most women treated with mastectomy with or without reconstruction can safely be followed by clinical exam alone or in conjunction with ultrasound. As long as the breast parenchyma has been completely removed during mastectomy, routine mammography on the affected side will not be neces- sary [16]. However it should be noted that some centers will ignore this advice and perform regular mammography in asymptomatic women after mastectomy with or without reconstruction [17]. If there is suspicion of significant residual breast parenchyma after mastectomy, breast MRI can reliably confirm or exclude residual parenchyma. Ipsilateral remaining breast tissue after mastectomy in women who have not received radiation therapy substantially increases the risk for local recurrence and may require re-excision or careful imaging surveillance [18]. In patients with mastectomy and implant reconstruction, MRI is superior to mammography and palpation regarding detection of recurrent disease [19]. MRI will be especially valuable in cases with prepectoral positioning of the implant to exclude pectoral recurrence behind the implant, as this area will not be accessible to clinical exam and ultrasound.

\subsubsection{Imaging Patients with Local Symptoms}

Any locoregional symptom such as a palpable lump, new pain, changes in breast configuration, or skin changes in a patient with a personal history of breast cancer should be carefully evaluated by a thorough clinical exam and tailored imaging. Many palpable abnormalities are readily accessible to targeted ultrasound. Additional imaging such as mammography and/or MRI can be added as needed. Positron emission tomography-computed tomography (PET/CT) can be very helpful in cases with suspected axillary recurrence, especially if the findings are not accessible to percutaneous biopsy.

\subsubsection{Strengths and Weaknesses of Different Surveillance Methods}

\subsubsection{Clinical Exam}

The value of a thorough clinical exam that consists of historytaking, inspection, and palpation of the breast and regional lymph nodes for the detection of local breast cancer recurrences should not be underestimated. Skin changes detected during clinical inspection may be the only sign of a local recurrence around the surgical scar or even for the existence of skin metastases. Palpation of the regional lymph nodes is also an important part of the clinical exam, especially if mammography only (no accompanying ultrasound) surveillance regimes are employed. About half of all ipsilateral breast recurrences will have a positive finding on clinical exam, and about a one-third of ipsilateral breast recurrences 
will be detected by the clinical exam with negative mammography [9]. This proportion is substantially higher than for healthy women undergoing routine mammography screening. Most guidelines therefore recommend clinical follow-up visits for breast cancer patients in the first 5 years after diagnosis at shorter intervals than the imaging surveillance (e.g., every 3-6 months) [3]. These visits also provide psychological support, motivate to follow adjuvant treatments recommendations, and help to detect treatment related problems early.

\subsubsection{Mammography}

Regular, usually annual mammography is the foundation of every surveillance regime in women after breast-conserving therapy. Ultrasound (US) and/or MRI can be used to supplement, but not to replace mammography in this situation. Between 50 and $80 \%$ of ipsilateral breast recurrences will be detectable by mammography. Key to this is the detection of suspicious microcalcifications by mammography, often an early sign of residual or recurrent disease (Fig. 14.1). High breast density as well as postoperative changes such as hematoma, seroma, fat necrosis, or scarring may reduce the sensitivity of mammography. There is hope that DBT will be able to overcome these limitations at least in part, by reducing problems due to superposition.

\section{Key Point}

- The detection of suspicious microcalcifications by mammography plays a crucial role in the early detection of not only in situ but also invasive ipsilateral breast cancer recurrence.

\subsubsection{Ultrasound}

Breast US is an ideal complementary technique to the clinical exam and mammography. It enables direct correlation with and work-up of clinical as well as mammographic findings. In addition, US is able to evaluate the chest wall or the reconstructed breast after mastectomy. Breast ultrasound is also an excellent tool to evaluate the regional lymph nodes. In contrast to mammography, the detection performance of US is not affected by dense breast tissue. The main limitation of US is the high number of non-specific or false-positive findings, although this is less of a problem if US is performed on a regular basis during follow-up.

\subsubsection{MRI of the Breast}

MRI of the breast has by far the highest sensitivity for invasive breast cancer of all available imaging modalities. This translates into a very high negative predictive value approaching $100 \%$ and makes MRI the ideal problem-solving tool. If no relevant contrast enhancement is found on MRI in the area of an equivocal clinical, mammographic, or sonographic finding, clinically relevant changes can be excluded with sufficient certainty, and the patient can safely be placed on short-term follow-up. With the exception of the immediate postoperative phase, MRI can also reliably differentiate between postoperative changes/scarring and recurrence. However, it is important to realize that local recurrence may lack typical malignant features at imaging such as irregular borders or rapid contrast uptake on MRI. Therefore histological confirmation, if necessary by open excisional biopsy, is advisable for all new solid lesions with enhancement on MRI (Fig. 14.2). The disadvantage of MRI is that many otherwise occult benign breast changes (including high-risk lesions) will also show enhancement on MRI. For MRI to have the highest benefit in routine surveillance/screening, the underlying incidence of malignant changes has to be high enough, or the positive predictive value of an abnormal MRI finding will be too low. This is the reason why routine surveillance with MRI is currently recommended only in the high-risk setting [15].

\section{Key Point}

- Any new solid enhancing lesion in the ipsilateral breast or chest wall should be considered suspicious for recurrence regardless of size, morphology, or enhancement characteristics.

\subsubsection{Other Imaging}

Contrast-enhanced mammography is an emerging technology, which may serve as an alternative if MRI cannot be performed (e.g., due to claustrophobia). Positron emission technologies such as PET/PEM are not recommended for routine surveillance of the breast due to the relatively high associated radiation dose associated with these modalities. However, PET/CT may be helpful in the evaluation of a possible regional lymph node recurrence or if there is the possibility of additional distant metastasis. 

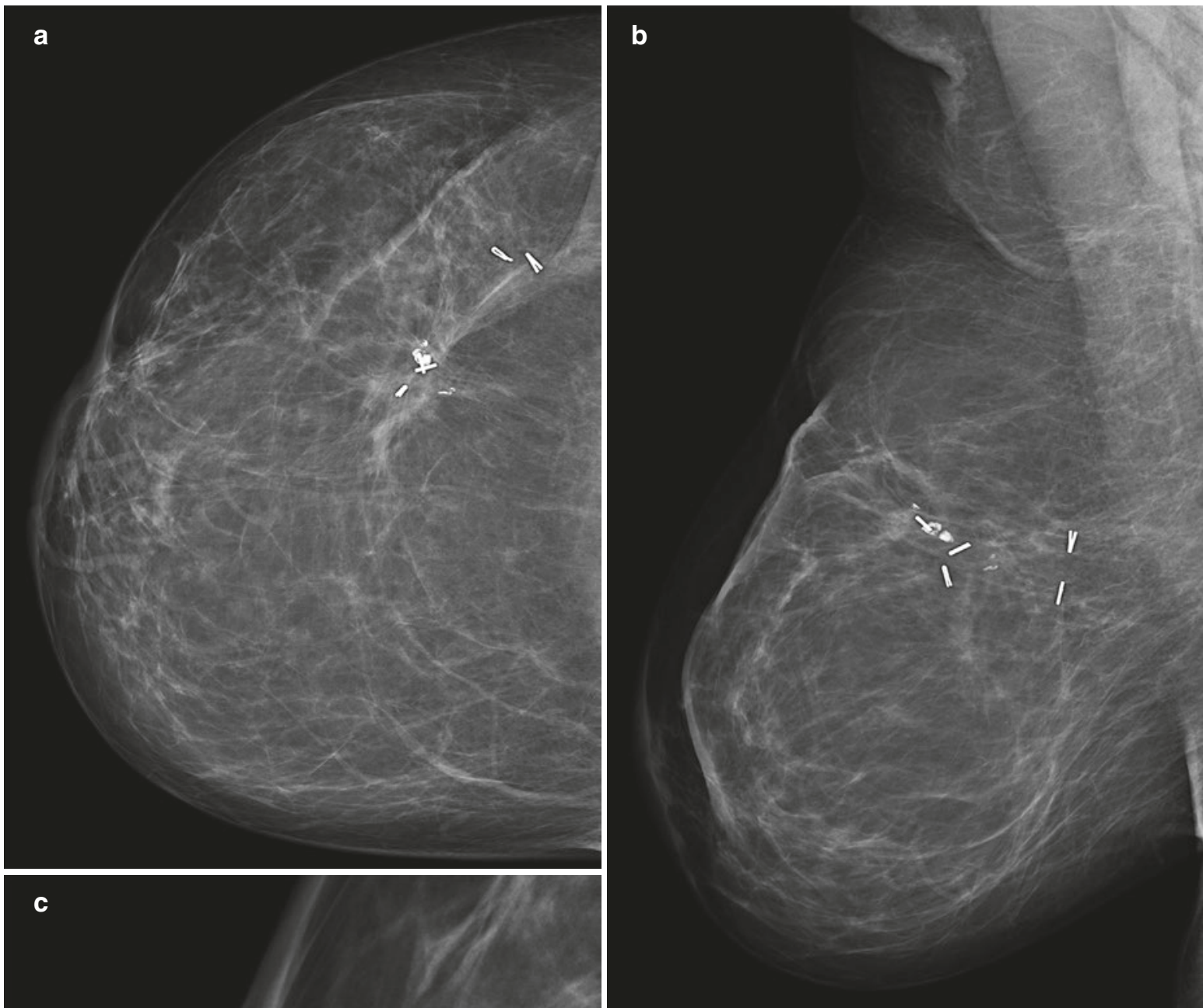

c

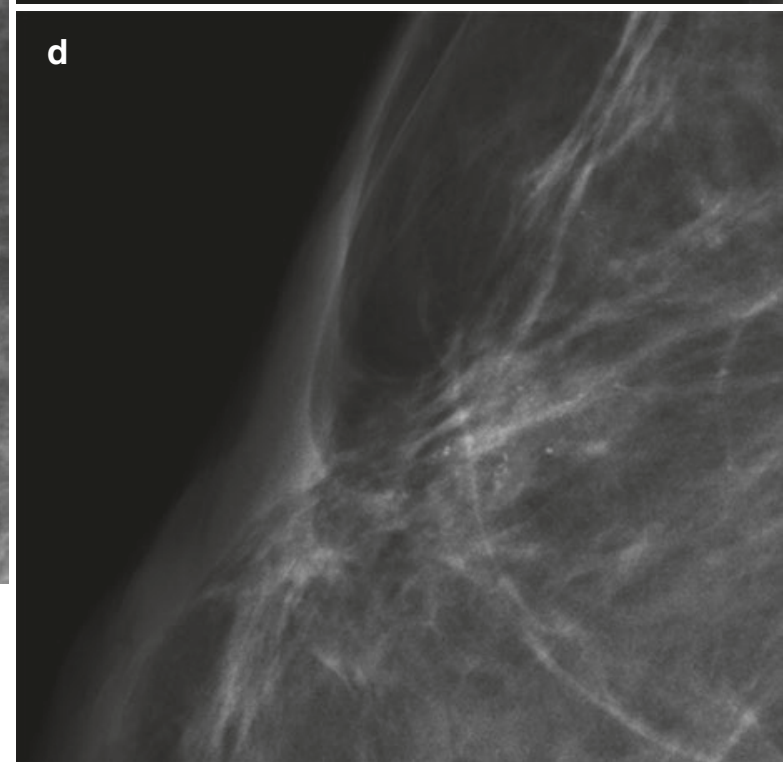

Fig. 14.1 A 48-year-old asymptomatic female, 11 years after breastconserving treatment for right breast cancer (invasive NST with associated DCIS high-grade, pN2, pN1a, G2, ER/PR positive, Her2 negative) treated with adjuvant chemotherapy and endocrine therapy under routine surveillance. CC (a) and MLO (b) views of the right breast. Enlarged views of the retroareolar region of the prior $\mathrm{CC} 1$ year ago (c) and the current $\mathrm{CC}(\mathbf{d})$ demonstrate a slight increase in the number of subtle calcifications in this area. Clinical exam and tailored ultrasound

were normal. Stereotactic vacuum-assisted biopsy with specimen radiography of the obtained cores (e) and post-biopsy views (f) to confirm correct clip placement was performed and revealed a poorly differentiated invasive breast cancer (NST). Mastectomy confirmed a 13-mm invasive breast cancer (rpT1c, rpN0, G3, ER 100\%, PR 100\%, Her2 negative, MIB-1 proliferation index 10\%). The patient was placed on tamoxifen and is currently healthy at age 53 

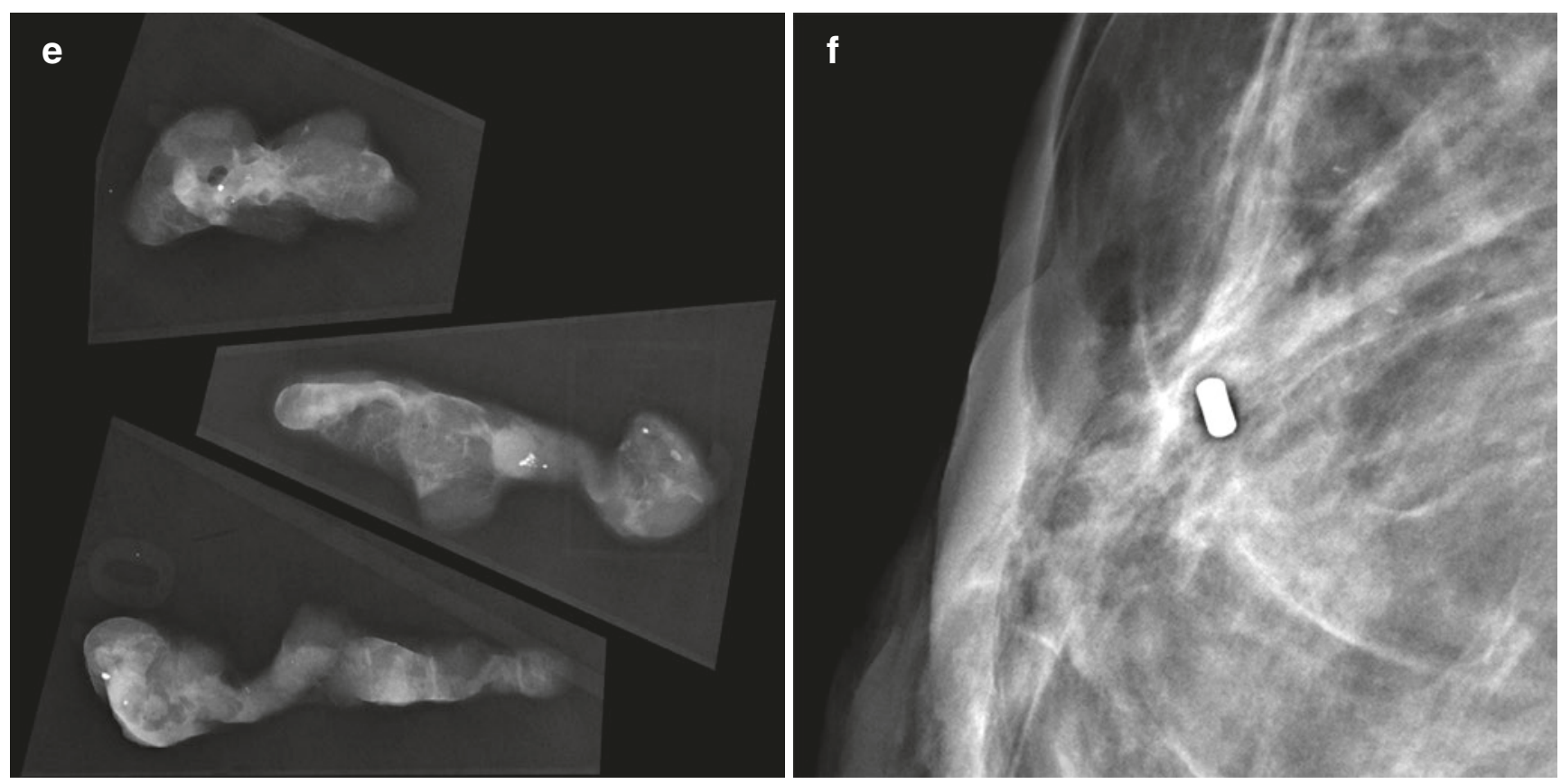

Fig. 14.1 (continued)
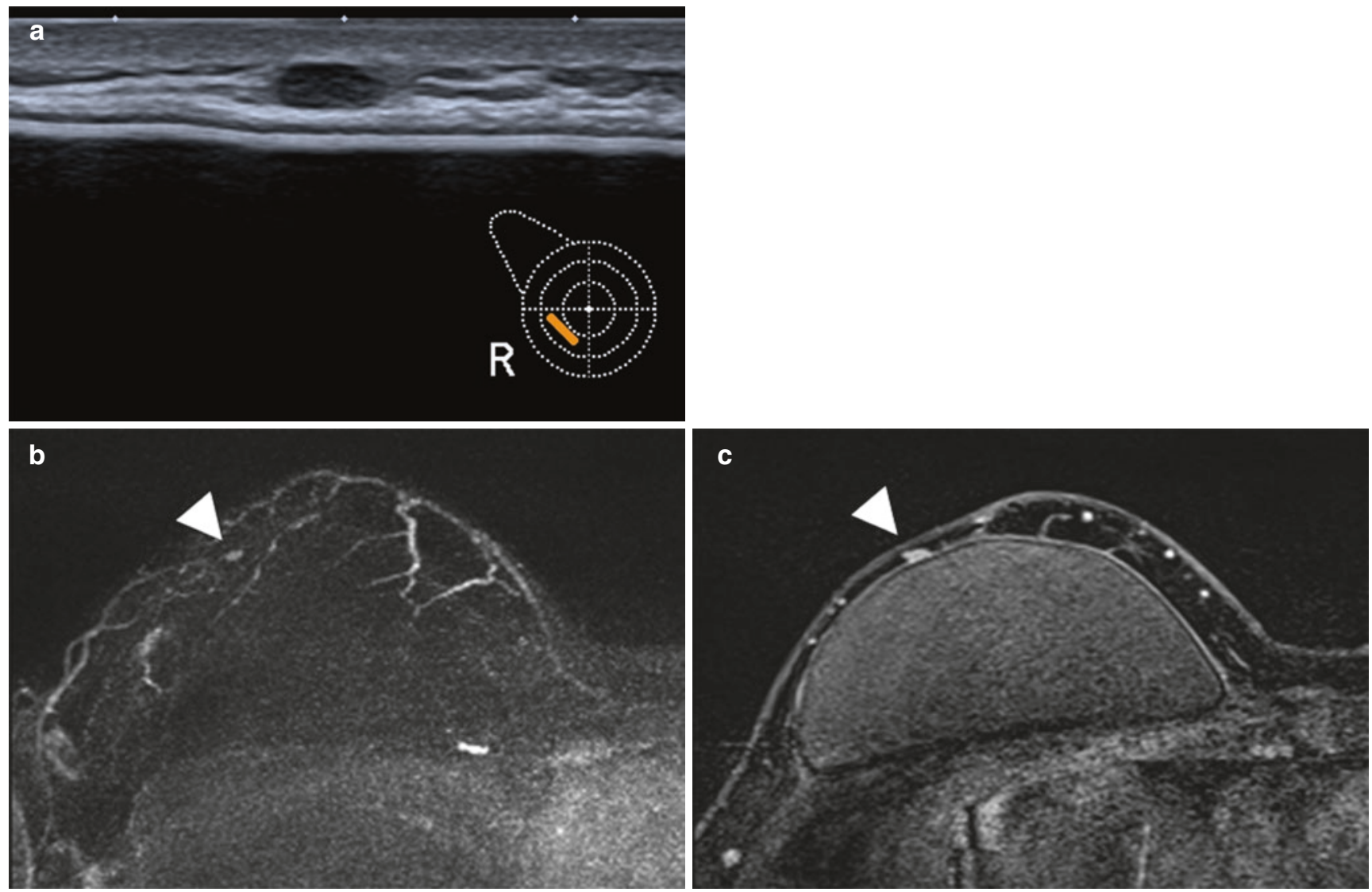

Fig. 14.2 A 37-year-old asymptomatic female, 6 years after right nipple-sparing mastectomy and implant reconstruction for extensive DCIS. Routine surveillance ultrasound (a) shows a new, small, oval, circumscribed, hypoechoic mass with parallel orientation in the remaining subcutaneous tissue surrounding the implant on the right. Contrastenhanced MRI for further evaluation was performed. MIP images in the second minute after contrast enhancement (b) and delayed images with

fat saturation (c) confirm a small, oval, circumscribed enhancing mass with persistent enhancement in the delayed phase. On surgical excision an invasive breast cancer (NST) with a maximum size of $6 \mathrm{~mm}$ was found (pT1b, pN0, G2, ER 100\%, PR 0\%, Her2 positive, MIB-1 proliferation index 30\%). The patient was treated with chemotherapy in combination with trastuzumab as well as tamoxifen and is currently healthy at age 40 


\subsection{Imaging of Distant Metastases}

\subsubsection{Initial Staging}

Once breast cancer is diagnosed, staging of the axilla must be performed. Currently, sentinel lymph node biopsy is recommended because imaging is not accurate enough [7]. Several guidelines recommend against the routine use of imaging modalities to stage asymptomatic metastases in newly diagnosed breast cancer [4]. Modern imaging technologies have improved the detection capability; thus the current NCCN guidelines [8] recommend against routine staging of patients with stage I and II disease but recommend CT (lung plus abdomen) or MRI (abdomen/liver) in addition to bone scintigraphy for stage III and IV disease. The use of FDG-PET/ CT is viewed with caution though several studies demonstrate more accurate breast cancer staging with PET/CT. The use of FDG-PET/CT changed the clinical stage in $30 \%$ and found unsuspected metastases in $21 \%$ of patients with stage IIA and IIIC disease. Particular in younger patients $(<40$ years of age), an upstaging of $17 \%$ was seen in stage IIB disease [20]. Even more in bone metastases FDG-PET/CT outperforms CT or bone scintigraphy, with accuracies of up to $90 \%$. The role of PET/CT may be less clinically relevant in extensive metastatic disease; however there are cases where a single metastasis is detected which affects the prognosis and the therapy (Fig. 14.3). The results of FDG-PET/CT are excellent; however it should be noted that its utility depends on cancer type being less sensitive in lobular breast cancer disease. Nevertheless it can be expected that PET/CT with more specific tracers than FDG will enhance the ability to stage breast cancer more accurately and will allow better prediction and assessment of treatment response during targeted therapy [7].

\section{Key Point}

- NCCN guidelines recommend against routine staging of patients with stage I and II disease but recommend CT or MRI in addition to bone scintigraphy for stage III and IV disease [8].

\subsubsection{Distant Metastases}

Distant recurrence (metastasis) is the main cause of breast cancer death. Mastectomy or lumpectomy followed by radiation therapy does not influence this risk [21]. The most common sites for breast cancer metastases are the skeleton, lung, liver, and brain [22]. In a Cochrane review by Rojas et al., which included 3055 women, there was no difference in overall or disease-free survival rates for patients who underwent intensive laboratory and imaging testing compared to those managed with clinical visits and mammography were seen
[22]. Similar results were observed in two multicentric randomized surveillance studies performed in Italy in asymptomatic breast cancer patients. In both studies patients were randomized in two groups. One group had intensive followup, including bone scintigraphy, chest x-ray, and ultrasound of the liver, whereas the control group was examined only with imaging and laboratory test when clinical symptoms were present. In both studies intensive testing found more metastases; however, there was no significant difference seen in the overall survival between the two groups [23, 24]. As a consequence $[3,5,6]$, several guidelines (Table 14.1) do not recommend intensive surveillance that would include routine blood tests, blood tests for tumor markers, chest X-ray, ultrasound of the liver, CT, MRI, or even PET/CT [5]. Nevertheless, surveillance programs vary among countries, organizations, and physicians. Physicians and patients favor intensive surveillance. In addition patients overestimate the role of laboratory and imaging tests and often incorrectly perceive the significance of a normal test [25, 26]. Keating et al. showed that patients who received care from oncologists had higher rates of testing than patients who were followed by their primary care physicians [25]. Grunfeld et al. [27] saw similar results were seen be in a retrospective study of 11,219 asymptomatic breast cancer survivors. Twenty-five percent of all patients had fewer than the recommended surveillance mammograms, and 50\% had more than the recommended surveillance for metastatic disease (including bone scans, chest x-ray, CT, or MRI). Higher morbidity (more mastectomies) and seeing both an oncologist and primary care physicians increased the odds of having more intense imaging testing. Other studies found similar results, with the overuse of blood tests although rising tumor markers (i.e., CA 15-3 and CEA) suggest recurrence in asymptomatic patients [28]. Recently several studies demonstrated that in breast cancer patients with rising CA 15-3 and CEA levels the use of FDG-PET/CT would be of high diagnostic value, reporting an accuracy of up to $92 \%$ [29, 30]. Although FDG-PET/CT is not recommended for follow-up, suspected cases of recurrence that have equivocal conventional studies would be the best candidate for PET/CT [29]. In a recent study, Parmar et al. [5] assessed the role of imaging modalities in follow-up programs. Surprisingly, only $55 \%$ of patients showed strict adherence to the surveillance program. During the study period (2011-2007), the use of bone scans and mammograms decreased (21\% to $13 \%$ and $81 \%$ to $75 \%$, respectively), whereas use of MRI and FDG-PET/CT increased significantly $(0.5 \%$ to $7 \%$ and $2 \%$ to $9 \%$, respectively).

Overall, when imaging and laboratory tests are ordered in asymptomatic patients during follow-up surveillance, the cost-effectiveness and accuracy of applying supplemental testing must be considered. In addition efforts should be made to a more intensively educate of physicians to reduce to use of non-guideline conforming surveillance testing. Nevertheless, it has been evidentially seen that physicians 

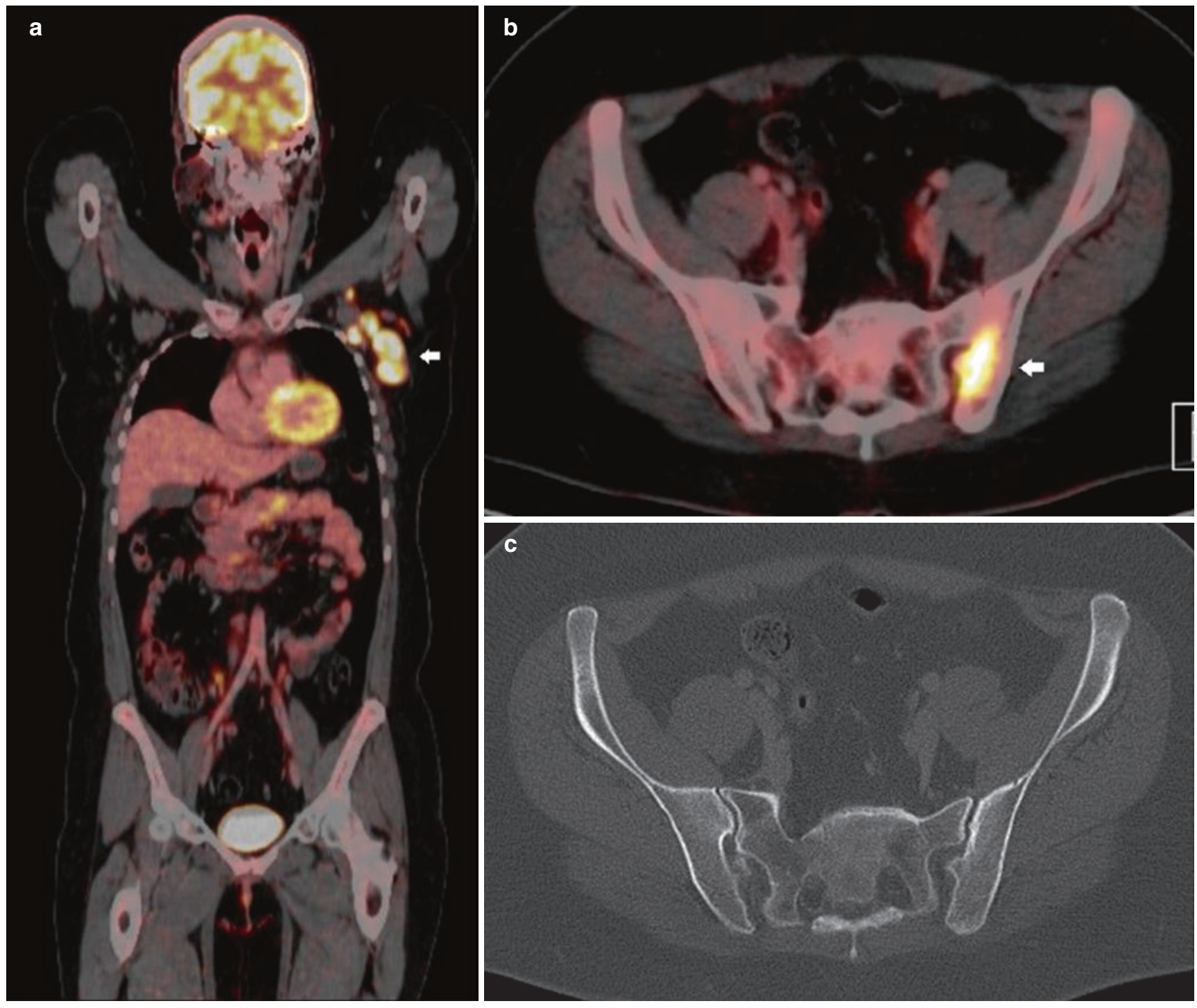

Fig. 14.3 A 41-year-old female with multicentric biopsy proven left breast cancer measuring $8 \mathrm{~cm}$ in dimensions and suspicious ipsilateral axillary nodes (stage T3 N1). Patient underwent 18F-FDG PET/CT for staging (histology: invasive ductal carcinoma NOS, G3, ER weakly positive, PR negative, HER2 positive, p53 weakly positive (10\%), MIB-1 positive). (a) Coronal fused PET/CT image shows bulky FDG avid left axillary nodes (arrow). (b) Axial fused PET/CT image shows a FDG avid lesion in the left ilium (arrow) and biopsy proven osseous metastases. This was occult on the CT component (c). PET/CT correctly upstaged the disease to stage IV thus illustrating the value of $\mathrm{PET} / \mathrm{CT}$ in distant staging and patients are not confident in the current surveillance programs with mammography as the only imaging modality considering the development of new and promising imaging technologies [29, 30]. Currently there is no evidence that early detection of metastases during follow-up improves survival. However, early detection may allow more accurate and curative intervention, and this may be the cause for the current trends, in which advanced imaging modalities other than mammography are used [5, 29].

\section{Key Point}

- Breast cancer patients who receive intensive screening and surveillance with imaging and laboratory studies have no survival benefit compared to those who undergo testing after clinical symptoms are evident. 


\subsubsection{Imaging for Bone, Lung, and Liver Metastases}

Current guidelines (Table 14.1) recommend against the routine use of laboratory or imaging tests to detect asymptomatic metastases during staging or follow-up after breast cancer diagnosis. This paragraph describes briefly which imaging modalities should be used to detect distant metastases if clinical symptoms are present [6]. To detect skeletal metastases, bone scintigraphy is more effective than conventional radiography. Several recent studies suggest that FDG-PET/CT, PET/MRI, or whole-body MRI is significantly more accurate than bone scintigraphy, but both can still miss some metastases, particularly sclerotic ones. It is expected that these new imaging techniques will replace current technologies (Fig. 14.4). To detect lung metastases chest $\mathrm{x}$-ray and CT are recommended. Chest $\mathrm{x}$-ray is considered the most reasonable approach because of the low costs. In case of any questionable findings, $\mathrm{CT}$ is recommended as a baseline for monitoring and for follow-up. Liver metastases are not as common as lung or bone metastases; however, their appearance is associated with the worst prognosis. In symptomatic patients CT or MRI is recommended as more lesions can be diagnosed with a higher accuracy compared to US. Particularly for follow-up during therapy MRI should be advantageously used. Breast cancer is second only to lung carcinoma as a cause of brain metastases. Contrast-enhanced MRI has largely replaced CT for the detection and evaluation of brain lesions because of its high sensitivity (Fig. 14.5) [6].

\subsubsection{Assessing Treatment Response of Distant Metastases}

Once a breast cancer patient has been diagnosed with metastases, accurate assessment of treatment response is of the uppermost importance. Currently follow-up assessment is based on anatomic imaging (CT or MRI) (Fig. 14.6). Treatment response, particularly in clinical trials, is evaluated by the RECIST (Response Evaluation Criteria in Solid Tumors) 1.1 criteria. A change in the size of a given metastasis during treatment is a measure of response. In soft tissue metastases (e.g., liver, lung, and brain), this approach is currently acceptable. However, in bone metastases - the most common site of metastatic disease - this approach is problematic unless there is an extra-osseous soft tissue mass associated with the bone lesion. If FDG-PET/CT is used metabolic activity can be assessed and thus determine the response to treatment [7, 29, 30]. By comparing FDG-PET/ CT with conventional anatomic CT, Vranjesevic et al. showed that the PPV (93\% vs. $85 \%$ ) and NPV (84\% vs. $59 \%$ ) of FDG-PET/CT were higher than with conventional imaging [7]. Other PET tracers (e.g., 18F-fluorothymidine) predict response at an early time point, and thus treatment change can be determined earlier which may lead to a better outcome with respect to disease burden and morbidity. Such approaches have been seen in lymphoma patients and should be implemented for breast cancer as well [7]. In addition PET/CT shows great promise in the assessment of patients who have various responses in different sites of metastases

Table 14.1 Surveillance recommendations for women treated for primary breast cancer

\begin{tabular}{|c|c|c|c|c|}
\hline & Year & $\begin{array}{l}\text { History and physical } \\
\text { examinations }\end{array}$ & Mammography (MG) & Other tests \\
\hline $\begin{array}{l}\text { American Society } \\
\text { of Clinical } \\
\text { Oncology }\end{array}$ & 2012 & $\begin{array}{l}\text { Every } 3-6 \text { month for } \\
\text { first } 3 \text { years } \\
\text { Every 6-12 months for } \\
\text { years } 4-5 \\
\text { Annual follow-up } \\
\text { thereafter }\end{array}$ & $\begin{array}{l}\text { Posttreatment MG } 1 \text { year } \\
\text { after initial mammogram } \\
\text { At least } 6 \text { months after } \\
\text { completion of radiation } \\
\text { therapy } \\
\text { Annual MG }\end{array}$ & $\begin{array}{l}\text { Chest radiography, bone scans, liver US, CT, PET, MRI, } \\
\text { or other laboratory tests: NOT recommended in } \\
\text { asymptomatic patients with no specific findings on } \\
\text { clinical examinations }\end{array}$ \\
\hline $\begin{array}{l}\text { National } \\
\text { Comprehensive } \\
\text { Cancer Network }\end{array}$ & 2018 & $\begin{array}{l}\text { Every } 4-6 \text { months for } \\
5 \text { years } \\
\text { Annual follow-up } \\
\text { thereafter }\end{array}$ & MG every 12 months & $\begin{array}{l}\text { MRI considered in women with lifetime risk of second } \\
\text { primary breast cancer greater than } 20 \% \\
\text { Imaging and laboratory tests not recommended in } \\
\text { asymptomatic patients }\end{array}$ \\
\hline $\begin{array}{l}\text { European Society of } \\
\text { Medical Oncology }\end{array}$ & 2013 & $\begin{array}{l}\text { Every } 3-4 \text { months for } \\
\text { first } 2 \text { years } \\
\text { Every } 6 \text { months from } \\
\text { year 3-5 } \\
\text { Annual follow-up } \\
\text { thereafter }\end{array}$ & $\begin{array}{l}\text { Ipsilateral (after BCS) and } \\
\text { contralateral MG every } \\
1-2 \text { years }\end{array}$ & $\begin{array}{l}\text { MRI may be indicated for young women with dense } \\
\text { breasts, genetic of familial predispositions } \\
\text { Laboratory or imaging tests not recommended in } \\
\text { asymptomatic patients }\end{array}$ \\
\hline $\begin{array}{l}\text { National Institute for } \\
\text { Clinical Excellence }\end{array}$ & 2011 & $\begin{array}{l}\text { Regular check-up, } \\
\text { determined by } \\
\text { physician or patient }\end{array}$ & Annual MG & Laboratory or imaging tests not recommended routinely \\
\hline
\end{tabular}

Note: Modified from [5]

$B C S$ breast-conserving surgery, $C T$ computed tomography, MRI magnetic resonance imaging, PET positron emission tomography, US ultrasonography 


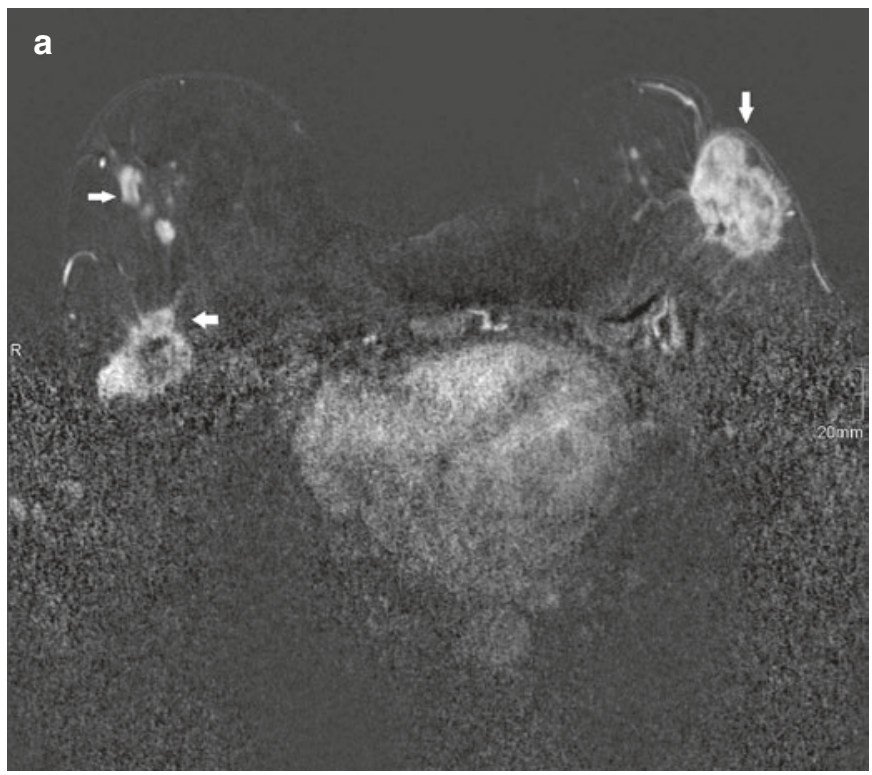

Fig. 14.4 A 66-year-old female with bilateral biopsy proven multicentric breast cancers underwent whole-body 18F-FDG PET/MRI which enables "one stop" staging of both locoregional and distant disease (histology: right invasive ductal carcinoma NOS, G2, ER/PR positive, HER2 negative, p53 weakly positive (10\%), MIB-1 20\% positive; left invasive ductal carcinoma NOS G2, ER/PR positive, HER2 weakly positive (20\%), p53 weakly positive (10\%), MIB-1 10\% positive). (a) Contrast-enhanced axial T1 subtracted breast MRI image shows multi-

due to tumor heterogeneity. Several studies have reported discordant responses between bone and non-bone metastases in 30-43\% [7]. Further FDG-PET/CT demonstrated response in patients who had no change at anatomic $\mathrm{CT}$ and predicted progression-free survival and disease-specific survival significantly better than CT [7].

\subsection{Concluding Remarks}

A well-defined evidence-based surveillance protocol is needed to manage patients with breast cancer after the initial diagnosis, as well as during staging and follow-up. The aims of any follow-up are to detect early local recurrence and to
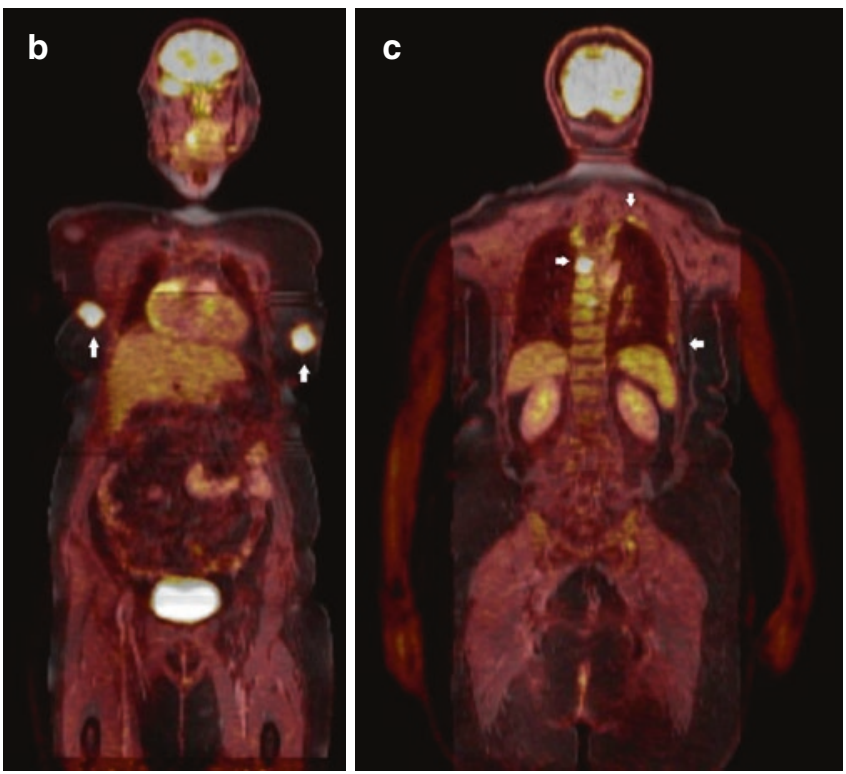

centric disease on the right (arrows) involving the skin, nipple, and chest wall and a dominant left breast mass (arrow) involving the nipple; bilateral T4 tumors $(\mathbf{b}, \mathbf{c})$ Coronal fused whole-body PET/MRI images show bilateral FDG avid breast tumors (arrows) (b) and multiple FDG avid osseous metastases (arrows) (c). PET/MRI also revealed bilateral FDG avid axillary and supraclavicular nodes (not shown). Overall stage T4, N3, M1

diagnose and treat cancer and/or therapy-related diseases such as metastases. Currently, mammography every 1-2 years is the only recommended evidence-based imaging modality. In asymptomatic patients, there are no data to indicate that any imaging or laboratory test leads to a survival benefit; thus these tests are not recommended during surveillance. Recent guidelines recommend CT or MRI in addition to bone scintigraphy for stage III and IV disease at the time of diagnosis. In the near future, it can be expected that sensitive imaging tests (whole-body MRI or PET/CT/MRI) will enhance the ability to stage breast cancer more accurately and will allow better prediction and assessment of treatment response during targeted therapy. 

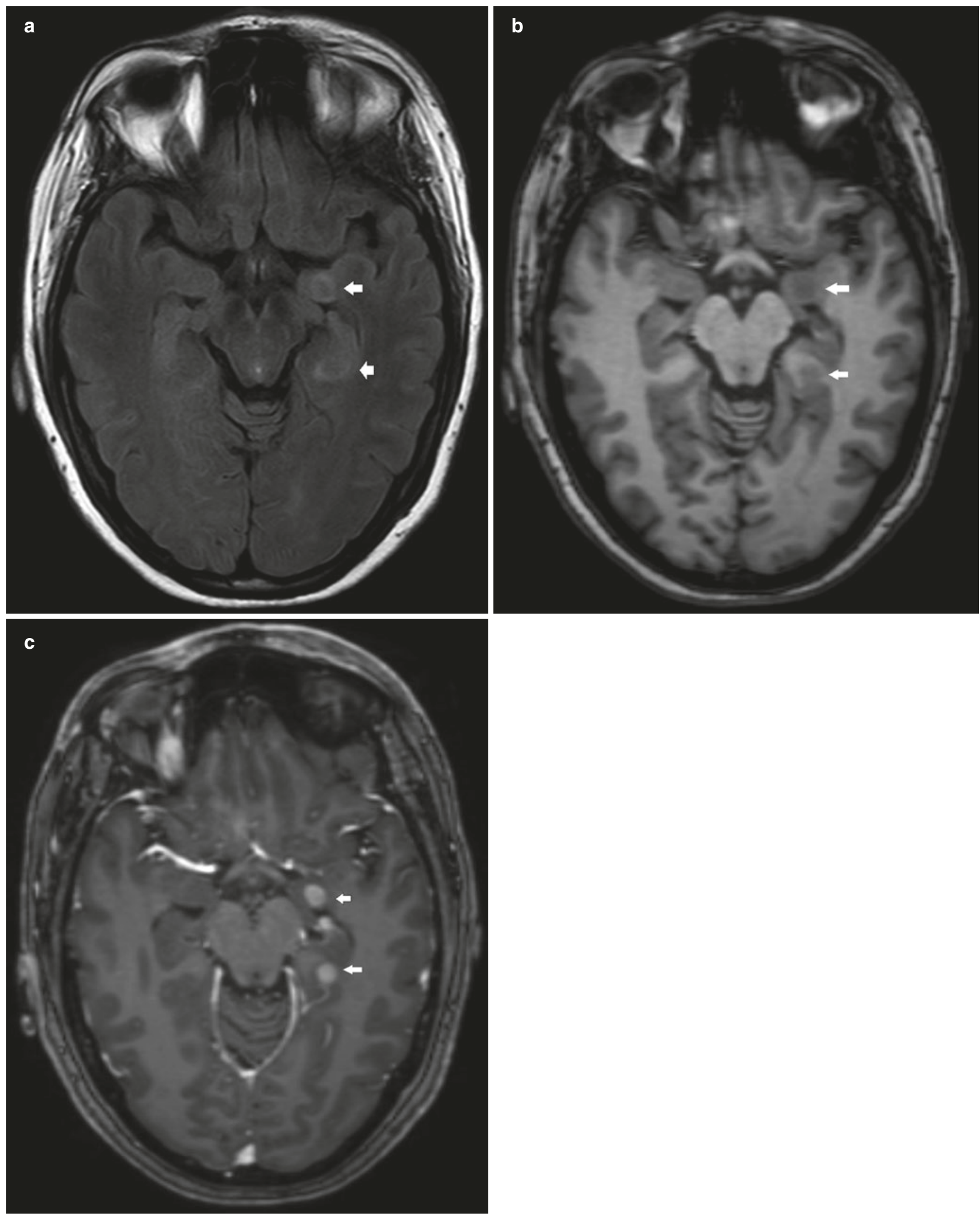

Fig. 14.5 A 40-year-old female with biopsy proven left breast cancer underwent contrast-enhanced brain MRI for further evaluation of headache (histology: invasive ductal carcinoma NOS, G2, ER positive, PR negative, HER2 positive, p53 negative, MIB-1 positive). Axial FLAIR

(a), T1(b) and post-contrast T1 (c) images show enhancing left temporal lesions in keeping with brain metastases which are heterogeneous predominantly hypointense on FLAIR and hypointense on T1 (arrows) 
Fig. 14.6 A 76-year-old female with biopsy proven right breast cancer and ipsilateral nodal disease (histology: invasive lobular carcinoma, G3, ER/PR negative, HER-2 positive, $\mathrm{p} 53$ positive, MIB-1 positive) with multiple mixed lytic and sclerotic osseous metastases in the spine and sternum on the sagittal CT bone window image (arrows) (a). Post chemotherapy the breast and nodal lesions regressed (not shown). On the postchemotherapy sagittal CT image (b), the osseous metastases (arrows) show interval increased sclerosis in keeping with posttreatment changes
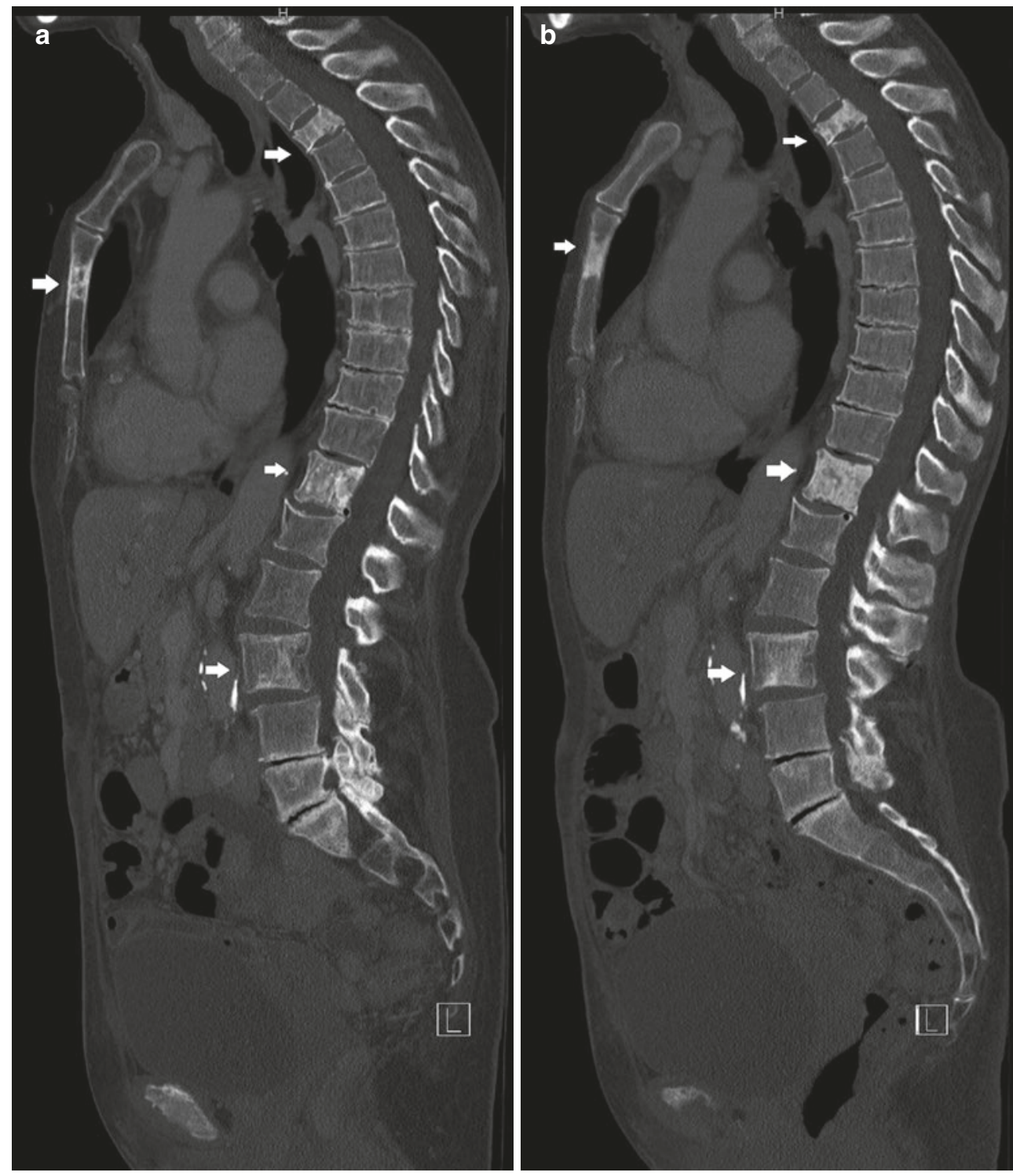

\section{Take-Home Messages}

- Regular, usually annual mammography is the cornerstone of surveillance of the ipsilateral breast in patients with a personal history of breast cancer who were treated with breast-conserving therapy.

- Local breast cancer recurrence may lack the typical imaging features of primary breast cancer, and reliable diagnosis is often based on the combination of a thorough clinical exam and tailored multimodality imaging.
- Current guidelines do not recommend intensive surveillance in asymptomatic breast cancer patients, which includes routine blood tests, chest x-ray, ultrasound of the liver, CT, MRI, or even PET/ CT. At time of diagnosis, CT or MRI in addition to bone scintigraphy is recommended in stage III and IV disease.

- More sensitive imaging tests (e.g., whole-body MRI or PET/CT/MRI) will enhance the ability to stage breast cancer more accurately and may change current guidelines. 


\section{References}

1. Siegel R, Naishadham D, Jemal A. Cancer statistics. CA Cancer J Clin. 2013;63:11-30.

2. Berry DA, Cronin KA, Plevritis SK, et al. Effect of screening and adjuvant therapy on mortality from breast cancer. N Engl J Med. 2005;353:1784-92.

3. Senkus E, Kyriakides S, Penault-Llorca F, et al. Primary breast cancer: ESMO Clinical Practice Guidelines for diagnosis, treatment and follow-up. Ann Oncol. 2013;24:vi7-vi23.

4. Brennan ME, Houssami N. Evaluation of the evidence on staging imaging for detection of asymptomatic distant metastases in newly diagnosed breast cancer. Breast. 2012;21:112-23.

5. Yoon JH, Kim MJ, Kim EK, Moon HJ. Imaging surveillance of patients with breast cancer after primary treatment: current recommendations. KJR. 2015;16:219-28.

6. Moy L, Bailey L, D'Orsi C, et al. ACR appropriateness criteria stage I breast cancer: initial workup and surveillance for local recurrence and distant metastases in asymptomatic women. J Am Coll Radiol. 2017;14:S282-92.

7. Jochelson M. Breast Cancer Staging. Physiology trumps anatomy. https://www.sbi-online.org/RESOURCES/WhitePapers/ TabId/595/ArtMID/1617/ArticleID/597/Breast-Cancer-StagingPhysiology-Trumps-Anatomy.aspx

8. National Comprehensive Cancer Network, Inc. 2018. The NCCN Evidence BlocksTM, NCCN Guidelines. Version 1.2018.

9. Houssami N, Ciatto S. Mammographic surveillance in women with a persona history of breast cancer: how accurate? How effective? Breast. 2010;19:439-45.

10. Spronk I, Schellevis FG, Burgers JS, et al. Incidence of isolated local breast cancer recurrence and contralateral breast cancer: a systematic review. Breast. 2018;39:70-9.

11. Lam DL, Houssami N, Lee JM. Imaging surveillance after primary breast cancer treatment. AJR. 2017;208:676-86.

12. Muradali D, Kennedy EB, Eisen A, et al. Breast screening for survivors of breast cancer: a systematic review. Prev Med. 2017;103:70-5.

13. Robertson C, Ragupathy SK, Boachie C, et al. Surveillance mammography for detecting ipsilateral breast tumour recurrence and metachronous contralateral breast cancer: a systematic review. Eur Radiol. 2011;21:2484-91.

14. Sia J, Moodie K, Bressel M, et al. A prospective study comparing digital breast tomosynthesis with digital mammography in surveillance after breast cancer treatment. Eur J Cancer. 2016;61:122-7.

15. Saslow D, Boetes C, Burke W, et al. American Cancer Society guidelines for breast screening with MRI as an adjunct to mammography. CA Cancer J Clin. 2007;57:75-89.

16. Khatcheressian JL, Hurley P, Bantug E, et al. Breast cancer followup and management after primary treatment: American Society of
Clinical Oncology clinical practice guideline update. J Clin Oncol. 2013;31:961-5.

17. Noroozian M, Carlson LW, Savage JL, et al. Use of screening mammography to detect occult malignancy in autologous breast reconstructions: a 15-year experience. Radiology. 2018;289:39-48.

18. Margolis NE, Morley C, Lotfi P, et al. Update on imaging of the postsurgical breast. Radiographics. 2014;34:642-60.

19. Bone B, Aspelin P, Isberg B, et al. Contrast-enhanced MR imaging of the breast in patients with breast implants after cancer surgery. Acta Radiol. 1995;36:111-6.

20. Riedl CC, Slobod E, Jochelson M, et al. Retrospective analysis of 18F-FDG PET/CT for staging asymptomatic breast cancer patients younger than 40 years. J Nucl Med. 2014;55:1578-83.

21. Buchholz TA, Hunt KK. Breast-conserving therapy: conventional whole breast irradiation. In: Harris JR, Lippman ME, Morrow M, Osborne CK, editors. Disease of the breast. Philadelphia, PA: Lippincott Williams \& Wilkins; 2010.

22. Rojas MP, Telaro E, Russo A, et al. Follow-up strategies for women treated for early breast cancer. Cochrane Database Syst Rev. 2005; 1:CD001768.

23. Rosselli Del Turco M, Palli D, Cariddi A, Ciatto S, Pacini P, Distante V. Intensive diagnostic follow-up after treatment of primary breast cancer. A randomized trial. National Research Council Project on Breast Cancer follow-up. JAMA. 1994;271:1593-7.

24. The GIVIO Investigators. Impact of follow-up testing on survival and health-related quality of life in breast cancer patients. A multicentre randomized controlled trial. JAMA. 1994;271:1587-92.

25. Keating NL, Landrum MB, Guadagnoli E, Winer EP, Ayanian JZ. Surveillance testing among survivors of early-stage breast cancer. J Clin Oncol. 2007;25:1074-81.

26. Loomer L, Brockschmidt JK, Muss HB, Saylor G. Postoperative follow-up of patients with early breast cancer. Patterns of care among clinical oncologists and a review of the literature. Cancer. 1991;67:55-60.

27. Grunfeld E, Hodgson DC, Del Giudice ME, Moineddin R. Population- based longitudinal study of follow-up care for breast cancer survivors. J Oncol Pract. 2010;6:174-81.

28. Chang HT, Hu C, Chiu YL, Peng NJ, Liu RS. Role of 2-[18F] fluoro-2-deoxy-D-glucose-positron emission tomography/computed tomography in the post-therapy surveillance of breast cancer. PLoS One. 2014;9:e115127.

29. Paydary K, Seraj SM, Zadeh MZ, et al. The evolving role of FDG$\mathrm{PET} / \mathrm{CT}$ in the diagnosis, staging, and treatment of breast cancer. Mol Imaging Biol. 2018; https://doi.org/10.1007/s11307-018-1181-3.

30. Champion L, Brain E, Giraudet AL, et al. Breast cancer recurrence diagnosis suspected on tumor marker rising: value of whole-body 18FDG-PET/CT imaging and impact on patient management. Cancer. 2011;117:1621-162.

Open Access This chapter is licensed under the terms of the Creative Commons Attribution 4.0 International License (http://creativecommons. org/licenses/by/4.0/), which permits use, sharing, adaptation, distribution and reproduction in any medium or format, as long as you give appropriate credit to the original author(s) and the source, provide a link to the Creative Commons license and indicate if changes were made.

The images or other third party material in this chapter are included in the chapter's Creative Commons license, unless indicated otherwise in a credit line to the material. If material is not included in the chapter's Creative Commons license and your intended use is not permitted by statutory regulation or exceeds the permitted use, you will need to obtain permission directly from the copyright holder. 